Methylation of the glucocorticoid receptor gene NR3C1: a summary for clinicians working with children and families

Victoria Brown

NHS Education for Scotland. NHS Grampian

doi: 10.1192/bjo.2021.643

Aims. It has been shown that the glucocorticoid receptor NR3C1 gene can be methylated ("switched off") in response to early adversity. Methylation has also been linked to physiological changes in the body's response to stress by changing the sensitivity of the hypothalamic-pituitary-adrenal (HPA) axis. In adults, associations have been made between NR3C1 methylation and borderline personality disorder, depression and post-traumatic stress disorder. Environmental and social co-variates increase with lifespan so establishing cause and effect is difficult. Studies in children, then, may illuminate patterns to inform current hypotheses.

This paper reviews the literature on children and adolescents linking glucocorticoid gene receptor NR3C1 to the psychopathology of mental illness. Findings are presented in an accessible manner to engage people less familiar with genetics and to inform frontline clinicians of this quickly growing area of research.

Method. MEDLINE and PsychINFO were searched for relevant peer-reviewed original research using the following keywords and associated mesh terms: NRC31, glucocorticoid receptor gene, methylation, epigenetics, child, adolescent, trauma, psychopathology, gene expression.

Result. 14 studies were identified involving 5475 young people. Degree of NR3C1 methylation was associated with severity of early life adversity. Methylation was linked with psychopathology including borderline personality disorder, internalising symptoms and externalising symptoms with sex differences. The most consistent association was with depression. Methylation seems to modulate the interaction between environment and genetics with the suggestion that the effect may be protective in some cases. However, longitudinal genetic sampling was only conducted in one study.

Conclusion. Heterogeneity of studies in the epigenetics field are discussed but should not detract from future possibilities. The hope is to identify therapeutic targets or monitor response to treatment as we work to better understand the biology of developmental psychology, mental illness and resilience. There is a growing understanding that epigenetic modifications likely change over time and clinical significance is most likely dictated by changes at multiple gene locations. Thus future research may need to move away from single gene research typically employed in favour of longitudinal whole genome studies in larger population studies.

It is time that clinicians integrate the concepts of "epigenetic adaptation to environmental stress" with "nature vs. nurture" in their psychoeducation with families. To understand that one's problems might be the symptom of environmental mismatch, and potentially reversible too, can bring validation and hope to families.

\section{Autistic and peudo-autistic traits in ongoing complex trauma}

Jennifer Bryden

Royal Cornhill Hospital, NHS Grampian, Tutor with PRIME

Partnerships in International Medical Education

doi: 10.1192/bjo.2021.644
Aims. To compare the neurodevelopmental profiles of Albanian street children to those predicted by the Coventry grid.

Background. A street children's centre had requested help to meet children's emotional needs. No program exists for children experiencing ongoing complex trauma. With input from widely-experienced consultant psychiatrist and consultant psychologist, a very low-intensity program of coping skills was piloted. Extensive anonymised notes were taken as part of the piloting.

The Coventry grid is a clinical tool comparing patterns of difficulties typically seen in autistic spectrum disorder (ASD) versus attachment difficulties. It's based on clinical experience and invites ongoing feedback.

Method. 12 Children aged 5-12 years completed the two-week program. The notes were examined for their relevance to areas of the Coventry Grid.

Result. The children showed both traits typical of ASD and of attachment problems. Identifying emotions was impossible for the youngest group (5-7 years); while the older groups could say whether someone was likely to feel "good" or "bad" but struggled to differentiate further.

Fantasy and symbolic play were hard for the younger children. If asked to imagine a situation, they replied "but that's not happening". One child constantly hugged a stuffed doll, but couldn't use it for play. Both younger groups found it hard to imagine a safe-place, though they could say what they wanted in it (chocolate and a working lightbulb). The oldest group all chose a real place related to the centre.

Generalising was difficult for all the children. The older children could say whether a story character was a good friend, but not apply this to real life. The youngest children were told a story about a dangerous stranger. Afterwards, the children said they would still go away with strangers as only the man in the story had said he wanted to harm children.

The younger children were diffusely attached, but the boys' eye contact, gesturing, and language were normal in all age groups. All children formed friendships easily, played in a group and were intensely loyal to siblings. They didn't show restricted interests, distress at changes to routine or sensory difficulties. They showed good awareness of the widely divergent social rules at the centre and at home.

Conclusion. The children showed a mix of traits usually associated with attachment difficulties and those usually associated with ASD. They may be different from UK clinic samples as they continued to experience severe trauma.

\section{Representation of \#CAMHS on social media platform TikTok}

Preetisha Chadee* and Sacha Evans

Great Ormond Street Hospital

${ }^{\star}$ Corresponding author.

doi: 10.1192/bjo.2021.645

Aims. The video-based free social media app, TikTok, has grown in popularity during the COVID-19 pandemic, with half of British children using Tik Tok regularly. With more than 2 billion downloads, it was the most downloaded app of 2020. Child and Adolescent Mental Health Services (CAMHS) is currently found on TikTok via the hashtag \#CAMHS. The aim of this study was to explore how CAMHS is represented on TikTok through reviewing the hashtags associated with CAMHS and exploring the themes of videos with the \#CAMHS hashtag.

Method. The Tik Tok app was downloaded and a search for the hashtags which featured the word \#CAMHS was undertaken. 